\title{
Theoretical and Practical Aspects of Estimation of Forced Sale Value ${ }^{* *}$
}

\begin{abstract}
This paper deals with the key categories used in the procedure of estimating the forced sale value of an object. We consider the different criteria and provide methodological recommendations on the classification of various objects of civil rights as objects of valuation. Materials of expert assessments and empirical observations are presented. A practice-oriented model for estimating liquidation value is proposed.
\end{abstract}

Keywords: valuation classification, liquidity, marketing period, implementation period of valuation aims

* Belarusian National Technical University, Minsk, Republic of Belarus

* The study was initiated by the author with the support of the Association of Valuation Organizations (Minsk, Republic of Belarus) 


\section{Forced Sale (Liquidation) Value as Definition in Theory of Property Valuation}

In the theory of estimating the value of objects of civil rights, the forced sale (liquidation) value usually means a kind of market value. It is used to implement some of the valuation aims that imply the reduction of the necessary and reasonably long marketing period required for the implementation of the 'classical' valuation aim the purchase and sale of property at market value [1-3]. Based on this definition, the marketing period characteristic for the formation of the forced sale value is shorter than necessary to reach the market value, and the conditions for marketing activities are worse. Accordingly, the forced sale value in absolute terms should be less than the market value. Thus, the essence of most of the developed methods for estimating the forced sale value is limited to the determination of the downward adjustment on the basis of different techniques and approaches (depending on the characteristics of the object of valuation itself) as well as the diverse micro- and macro-economic factors affecting the forced sale value [4-7].

\section{Practical Aspects of Estimating Forced Sale Value}

In terms of the practice of valuation across Belarus, for example, the national valuation standard provides for 23 basic valuation aims applicable to permanent structures [8]. The following three of them require the determination of the forced sale value:

1) public sale (at auction and by tender), including in a bankruptcy procedure (the forced sale value as the initial price at the auction can be considered as a kind of valuation base, but by the creditor's decision);

2) a pledge/mortgage may be considered in the case of sale when it comes to the failure to fulfill the loan recipient's obligations;

3) the sale of property seized, arrested, or forfeited to the state under the law on enforcement proceedings.

The result of the independent valuation (valuation report) is valid for a 6-month period with respect to the second one and for a 12-month period as regards the first and third of these three valuation aims. There are some other valuation aims and subject property; with regard to these, such a period lasts 12 months. In the previous versions of the standards, there were 6-, 9-, and 12-month periods. It was assumed that these values should be considered as the time frame for achieving or implementing a valuation aim; however, obvious is the fact that this contradicts both theory and practice. After all, the marketing period necessary for completing a purchase and sale transaction for quite a large number of valuation objects may actually be shorter. In this case, there is no point in adjusting for liquidity. 
Meanwhile, in practice, users of independent valuation such as 1) bank collateral services, 2) bailiff services, 3) auction companies, and 4) auction departments within the valuation organizations may have their own 'internal' standards applicable to the periods of selling objects of valuation (the time frame for the implementation of the valuation aim by the user of an independent valuation service). Interactions with them have shown that, in most cases, such users are guided by a one-month marketing period necessary for achieving their valuation aims, and in some exceptional situations by a period of up to three months. Furthermore, in the event of an auction, the Belarusian legislation even outlines the minimum one-month marketing period applicable to the object of valuation in the form of an open offer. Thus, in practice, there may be disagreements between the regulatory norms in the sphere of independent valuation and the legal field in the areas of the activities of the user of valuation.

The aforementioned contradictions may be resolved through indicating the estimated period of the client's valuation aim in the valuation assignment with respect to a particular object of valuation as well as introducing such a term as valuation aim implementation time (or period) into theory and practice.

\section{Liquidity of Real Estate: Criteria and Parameters of Classification}

In a number of cases, it was initially intended to use the forced sale value as the basis for valuation in implementing the above-mentioned valuation aims. However, it should be understood that there will be a small group of valuation objects to which the adjustment will not apply, because their normal marketing period will be shorter or comparable to the period of implementing the valuation aim. Therefore, to our mind, it is possible to distinguish the following levels of the liquidity of objects in terms of the appropriateness of making adjustments and their probable values (Fig. 1):

1.1. Extra high liquidity objects (the marketing period for a transaction at market value will roughly range from two weeks to one month).

1.2. High liquidity objects of valuation (the marketing period is approximately from one to six months).

1.3. Liquid objects (the marketing period is approximately from 6 to 18 months).

2. Medium liquidity objects (the marketing period is approximately from 18 to 36 months.

3. Low liquidity objects (the marketing period is approximately 36 months or more).

4. Non-liquid objects (in accordance with the original functional purpose, sale is unlikely for various reasons).

Note: approximate ranges of marketing periods are given for a balanced market under the conditions of the Republic of Belarus. 

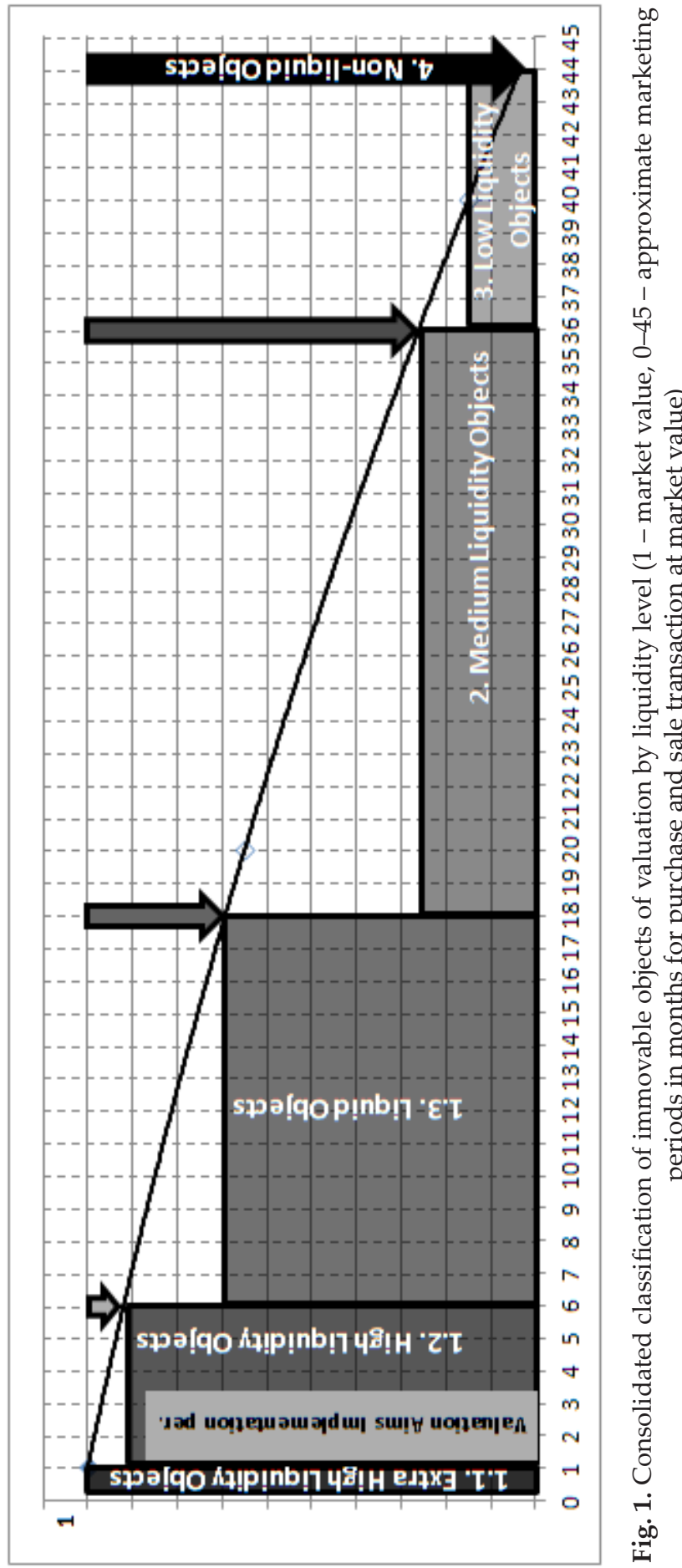
For the allocation of specific real estate objects with different functional purposes and parameters to the above groups, the classification shown in Table 1 can be used.

Table 1. The consolidated classification of real estate objects by liquidity levels; approximate typical marketing periods (intervals) for making purchase and sale transactions at market value, minimum liquidity adjustments for forced sale within a period of one to three months

(for the economic conditions of the Republic of Belarus as of Q1-Q2 2018)

\begin{tabular}{|c|c|c|c|c|c|}
\hline \multirow[b]{2}{*}{$\begin{array}{l}\text { Group } \\
\text { number }\end{array}$} & \multirow[b]{2}{*}{$\begin{array}{l}\text { Sub- } \\
\text { group } \\
\text { number }\end{array}$} & \multicolumn{4}{|c|}{ Classification criteria } \\
\hline & & \multirow{2}{*}{$\begin{array}{l}\text { Qualitative characteristic: } \\
\text { functional purpose } \\
3\end{array}$} & \multicolumn{3}{|c|}{$\begin{array}{c}\text { Quantitative } \\
\text { characteristic: size of } \\
\text { operational parameters }\end{array}$} \\
\hline 1 & 2 & & 4 & 5 & 6 \\
\hline 1 & \multicolumn{5}{|c|}{ Level (class) 1. Liquid 'universal' real estate objects } \\
\hline \multirow{5}{*}{1.1.} & \multicolumn{5}{|c|}{ Residential urban and suburban, non-residential urban real estate } \\
\hline & \multicolumn{5}{|c|}{$\begin{array}{l}\text { Sublevel 1.1. Extra high liquidity objects } \\
\text { (consolidated for sublevel: } 2 \text { weeks }-1 \text { month or more) }\end{array}$} \\
\hline & 1.1.1 & $\begin{array}{l}\text { One-room flat: } \\
\text { from } 2 \text { weeks to } 2 \text { months; } 2-5-7 \%\end{array}$ & small & medium & high \\
\hline & 1.1 .2 & $\begin{array}{l}\text { Two-room flat: } \\
1-4 \text { months; } 5-7-10 \%\end{array}$ & small & medium & high \\
\hline & 1.1 .3 & $\begin{array}{l}\text { Three-room flat: } \\
3-6 \text { months; } 10-15 \%\end{array}$ & small & medium & high \\
\hline \multirow{10}{*}{1.2 . } & \multicolumn{5}{|c|}{$\begin{array}{l}\text { Sublevel 1.2. High liquidity objects } \\
\text { (consolidated for sublevel: 1-6 months or more) }\end{array}$} \\
\hline & 1.2 .1 & $\begin{array}{l}\text { Four-room flat: } \\
1-6 \text { months; } 15-20-30 \%\end{array}$ & small & medium & high \\
\hline & 1.2.2 & $\begin{array}{l}\text { Five-room flat: } \\
6-24 \text { months; } 20-30 \%\end{array}$ & small & medium & high \\
\hline & 1.2 .3 & $\begin{array}{l}\text { VIP real estate: multi-room/multi-level flat: } \\
6-12 \text { months; } 20-40 \%\end{array}$ & - & medium & $\begin{array}{l}\text { high } \\
\text { (very } \\
\text { high) }\end{array}$ \\
\hline & \multicolumn{5}{|c|}{ Residential suburban real estate } \\
\hline & 1.2 .4 & $\begin{array}{l}\text { Cottage: } \\
\text { 6-24 months; } 20-40 \%\end{array}$ & small & medium & high \\
\hline & 1.2 .5 & $\begin{array}{l}\text { Townhouse: } \\
\text { 6-12 months; } 10-15 \%\end{array}$ & small & medium & high \\
\hline & 1.2 .6 & $\begin{array}{l}\text { House: } 3-9 \text { months; } \\
10-15-20 \%\end{array}$ & small & medium & high \\
\hline & 1.2 .7 & $\begin{array}{l}\text { Holiday house: } \\
\text { 3-12 months; } 15-30-40 \%\end{array}$ & small & medium & high \\
\hline & 1.2 .8 & $\begin{array}{l}\text { Land parcel: } \\
6-12 \text { months; } 5-20 \%\end{array}$ & small & medium & high \\
\hline
\end{tabular}


Table 1. cont.

\begin{tabular}{|c|c|c|c|c|c|}
\hline \multirow[b]{2}{*}{$\begin{array}{l}\text { Group } \\
\text { number }\end{array}$} & \multirow[b]{2}{*}{$\begin{array}{l}\text { Sub- } \\
\text { group } \\
\text { number }\end{array}$} & \multicolumn{4}{|c|}{ Classification criteria } \\
\hline & & \multirow{2}{*}{$\begin{array}{l}\text { Qualitative characteristic: } \\
\text { functional purpose } \\
3\end{array}$} & \multicolumn{3}{|c|}{$\begin{array}{c}\text { Quantitative } \\
\text { characteristic: size of } \\
\text { operational parameters }\end{array}$} \\
\hline 1 & 2 & & 4 & 5 & 6 \\
\hline \multirow{6}{*}{1.3 . } & \multicolumn{5}{|c|}{$\begin{array}{l}\text { Sub-level 1.3. Liquid objects } \\
\text { (consolidated for sublevel: } 6-18 \text { months or more) }\end{array}$} \\
\hline & \multicolumn{5}{|c|}{ Residential urban - real estate } \\
\hline & 1.3.1 & $\begin{array}{l}\text { Room in a flat: } \\
12-18 \text { months; } 30-80 \%\end{array}$ & small & medium & high \\
\hline & \multicolumn{5}{|c|}{ Non-residential urban real estate } \\
\hline & 1.3 .2 & $\begin{array}{l}\text { Garage: } \\
6-18 \text { months; } 15-30-40 \%\end{array}$ & small & medium & high \\
\hline & 1.3 .3 & $\begin{array}{l}\text { Parking space: } \\
\text { 6-24 months; } 25-55 \%\end{array}$ & \multicolumn{2}{|c|}{ outdoor } & indoor \\
\hline 2 & \multicolumn{5}{|c|}{$\begin{array}{l}\text { Level (class) 2. 'Special' objects of } \\
\text { medium liquidity (approximately 18-36 months or more) }\end{array}$} \\
\hline \multirow{4}{*}{2.1.} & \multicolumn{5}{|c|}{ Commercial real estate for administrative purposes } \\
\hline & 2.1.1 & $\begin{array}{l}\text { Office premises (with regard to the level of } \\
\text { equipment and location): } \\
6-24 \text { months; } 2-40 \%\end{array}$ & small & medium & high \\
\hline & 2.1 .2 & $\begin{array}{l}\text { Non-residential premises for administrative } \\
\text { purposes (storeroom, utility or process pre- } \\
\text { mises, small warehouse, etc.): } \\
6-24 \text { months; } 20-30 \%\end{array}$ & small & medium & high \\
\hline & 2.1 .3 & $\begin{array}{l}\text { Parking space and parking lots (outdoor and } \\
\text { indoor): } \\
\text { 18-36 months; } 25-35-50 \%\end{array}$ & small & medium & high \\
\hline \multirow{5}{*}{2.2 . } & \multicolumn{5}{|c|}{ Commercial property for sale } \\
\hline & 2.2.1 & $\begin{array}{l}\text { Shop: } \\
\text { 6-12 months; } 15-25 \%\end{array}$ & small & medium & high \\
\hline & 2.2 .2 & $\begin{array}{l}\text { Kiosk: } \\
6-36 \text { months; } 15-25 \%\end{array}$ & small & medium & high \\
\hline & 2.2 .3 & $\begin{array}{l}\text { Small trading premises, space: } \\
6-12 \text { months; } 20-30 \%\end{array}$ & small & medium & high \\
\hline & 2.3 .4 & $\begin{array}{l}\text { Shopping and entertainment center, trade } \\
\text { gallery: } \\
\text { 12-18 months; } 20-30 \%\end{array}$ & small & medium & high \\
\hline
\end{tabular}


Table 1. cont.

\begin{tabular}{|c|c|c|c|c|c|}
\hline 1 & 2 & 3 & 4 & 5 & 6 \\
\hline \multirow{4}{*}{2.3 . } & \multicolumn{5}{|c|}{ Commercial warehouse property } \\
\hline & 2.3 .1 & $\begin{array}{l}\text { Universal warehouse (not equipped): } \\
12-24 \text { months; } 45-55 \%\end{array}$ & small & medium & high \\
\hline & 2.3.2 & $\begin{array}{l}\text { Warehouse equipped: } \\
6-18 \text { months; } 25-35 \%\end{array}$ & small & medium & high \\
\hline & 2.3 .3 & $\begin{array}{l}\text { Warehouse - logistics center: } \\
6-12 \text { months; } 35-45 \%\end{array}$ & small & medium & high \\
\hline \multirow{11}{*}{2.4 . } & \multicolumn{5}{|c|}{$\begin{array}{c}\text { Manufacturing (industrial) real estate } \\
\text { (without technological machines and equipment) }\end{array}$} \\
\hline & 2.4 .1 & $\begin{array}{l}\text { Objects of agricultural purpose (livestock, } \\
\text { poultry production, etc.): } \\
18-36 \text { months; } 60-70-80 \%\end{array}$ & small & medium & high \\
\hline & 2.4.2. & $\begin{array}{l}\text { Mechanical engineering objects: } \\
18-36 \text { months; } 50-60-70 \%\end{array}$ & small & medium & high \\
\hline & 2.4 .3 & $\begin{array}{l}\text { Objects of the motor industry: } \\
18-36 \text { months; } 50-60-70 \%\end{array}$ & small & medium & high \\
\hline & 2.4 .4 & $\begin{array}{l}\text { Objects of the food industry: } \\
18-36 \text { months; } 50-60 \%\end{array}$ & small & medium & high \\
\hline & 2.4 .5 & $\begin{array}{l}\text { Objects of the construction industry: } \\
18-36 \text { months; } 50-60-70 \%\end{array}$ & small & medium & high \\
\hline & 2.4 .6 & $\begin{array}{l}\text { Objects of other spheres of industry: } \\
\text { 9-36 months; } 30-70 \%\end{array}$ & small & medium & high \\
\hline & 2.4 .7 & $\begin{array}{l}\text { Land parcels of agricultural purpose: } \\
\text { no data currently available }\end{array}$ & small & medium & high \\
\hline & 2.4 .8 & $\begin{array}{l}\text { Forest land plots: } \\
\text { no data currently available }\end{array}$ & small & medium & high \\
\hline & 2.4 .9 & $\begin{array}{l}\text { Fruit-and-berry gardens: } \\
\text { no data available now }\end{array}$ & small & medium & high \\
\hline & 2.4 .10 & $\begin{array}{l}\text { Open-cast mines, boreholes, mines: } \\
\text { no data are currently available }\end{array}$ & small & medium & high \\
\hline \multirow{4}{*}{2.5 . } & \multicolumn{5}{|c|}{$\begin{array}{l}\text { Real estate in services sector, small and medium enterprises } \\
\text { (without technological machines and equipment) }\end{array}$} \\
\hline & 2.5 .1 & $\begin{array}{l}\text { Objects (stations) of car service, car wash: } \\
9-18 \text { months; } 35-45-55 \%\end{array}$ & small & medium & high \\
\hline & 2.5 .2 & $\begin{array}{l}\text { Hair studios, salons: } \\
\text { 9-24 months; } 20-50 \%\end{array}$ & small & medium & high \\
\hline & 2.5 .3 & $\begin{array}{l}\text { Repair shops: } \\
\text { 6-12 months; } 20-30 \%\end{array}$ & small & medium & high \\
\hline
\end{tabular}


Table 1. cont.

\begin{tabular}{|c|c|c|c|c|c|}
\hline \multirow{3}{*}{$\begin{array}{c}\text { Group } \\
\text { number }\end{array}$} & \multirow{3}{*}{\begin{tabular}{|c|}
$\begin{array}{c}\text { Sub- } \\
\text { group } \\
\text { number }\end{array}$ \\
2 \\
\end{tabular}} & \multicolumn{4}{|c|}{ Classification criteria } \\
\hline & & \multirow{2}{*}{$\begin{array}{l}\text { Qualitative characteristic: } \\
\text { functional purpose } \\
3\end{array}$} & \multicolumn{3}{|c|}{$\begin{array}{c}\text { Quantitative } \\
\text { characteristic: size of } \\
\text { operational parameters }\end{array}$} \\
\hline & & & 4 & 5 & 6 \\
\hline \multirow{9}{*}{$\begin{array}{c}2.5 . \\
\text { cont. }\end{array}$} & 2.5 .4 & $\begin{array}{l}\text { Agricultural estates: } \\
\text { 12-36 months; } 30-70 \%\end{array}$ & small & medium & high \\
\hline & 2.5 .5 & $\begin{array}{l}\text { Parks: } \\
\text { no data are currently available }\end{array}$ & small & medium & high \\
\hline & 2.5 .6 & $\begin{array}{l}\text { Aqua zones: } \\
\text { no data are currently available }\end{array}$ & small & medium & high \\
\hline & 2.5 .7 & $\begin{array}{l}\text { Cafes (including roadside cafes), restau- } \\
\text { rants: } \\
9-36 \text { months; } 20-30 \%\end{array}$ & small & medium & high \\
\hline & 2.5 .8 & $\begin{array}{l}\text { Hotels (with regard to the level of equip- } \\
\text { ment and location), hostels, motels: } \\
24-36 \text { months; } 50-60 \%\end{array}$ & small & medium & high \\
\hline & 2.5 .8 & $\begin{array}{l}\text { Sports facilities (halls, stadiums, race tracks, } \\
\text { etc.): } \\
\text { no data are currently available }\end{array}$ & small & medium & high \\
\hline & 2.5 .9 & $\begin{array}{l}\text { Exhibition complexes, museums: } \\
\text { no data are currently available }\end{array}$ & small & medium & high \\
\hline & 2.5 .10 & $\begin{array}{l}\text { Healthcare Objects: } \\
\text { 6-12 months; 30-60\% }\end{array}$ & small & medium & high \\
\hline & 2.5 .11 & $\begin{array}{l}\text { Other service facilities (cinemas, casinos, etc.): } \\
6-36 \text { months; } 20-80 \%\end{array}$ & small & medium & high \\
\hline 3. & \multicolumn{5}{|c|}{$\begin{array}{l}\text { Level (class) 3. Special-purpose real estate objects } \\
\text { of low liquidity (approximately 18-36 months or more) }\end{array}$} \\
\hline & \multicolumn{5}{|c|}{ Objects of valuation of historical and cultural value } \\
\hline 3.1. & 3.1 & $\begin{array}{l}\text { Objects of religious cults, castles, fortresses, } \\
\text { bridges, homesteads, etc.: } \\
\text { no data are currently available }\end{array}$ & small & medium & high \\
\hline \multirow{3}{*}{3.2} & \multicolumn{5}{|c|}{$\begin{array}{c}\text { Special-purpose real estate, } \\
\text { not specified in the general classification }\end{array}$} \\
\hline & 3.2 .1 & $\begin{array}{l}\text { Not completed construction objects of the } \\
\text { group 1.1.1-2.5.11 and others: } \\
18-36 \text { months; } 50-70 \%\end{array}$ & small & medium & high \\
\hline & 3.2 .2 & $\begin{array}{l}\text { Other special-purpose objects: } \\
18-36 \text { months and more; } \\
20-70 \%\end{array}$ & small & medium & high \\
\hline
\end{tabular}


Table 1. cont.

\begin{tabular}{|c|c|c|c|c|c|}
\hline 1 & 2 & 3 & 4 & 5 & 6 \\
\hline 4. & \multicolumn{5}{|c|}{$\begin{array}{l}\text { Level (class) } 4 \text {. Non-liquid objects (in accordance with original functional purpo- } \\
\text { se, sale is unlikely for various reasons, } 18-36 \text { months or more) }\end{array}$} \\
\hline 4.1 & 4.1 & $\begin{array}{l}\text { Objects of levels } 1-3 \text { in critical condition: } \\
18-36 \text { months and more; } \\
20-80 \%\end{array}$ & small & medium & high \\
\hline 4.2 & 4.2 & $\begin{array}{l}\text { Real estate objects of unspecified functional } \\
\text { purpose: } \\
18-36 \text { months and more; } \\
20-80 \%\end{array}$ & small & medium & high \\
\hline
\end{tabular}

Source: author's material, source [8]; with the author's revision, sources [6, 7]

\section{Conclusions}

The classification of the real estate objects developed and presented in Table 1 allows an appraiser to fill the unknown intermediate positions by interpolating or extrapolating values to objects of different levels and liquidity sub-levels on the basis of the information on liquidity adjustments for specific positions.

The correlation between the marketing period intervals given in the table and the customer-specified timing of the valuation aim implementation is also a condition that will help increase the reliability of the calculation of the final forced sale value.

\section{References}

[1] Mezhdunarodnyye standarty otsenki. 6-e izdaniye 2003 (MSO 2003) - International Valuation Standards. Perevod Rossiyskogo obshchestva otsenshchikov, Komitet po Mezhdunarodnym standartam otsenki (IVSC), Moskva 2004 [Международные стандарты оценки. 6-е издание 2003 (МСО 2003) - International Valuation Standards. Перевод Российского общества оценщиков, Комитет по Международным стандартам оценки (IVSC), Москва 2004].

[2] Yevropeyskiye standarty otsenki. 8-ye izdaniye, 2016 - European Valuation Standards. Perevod Rossiyskogo obshchestva otsenshchikov, Yevropeyskaya gruppa otsenochnykh ob"yedineniy (TEGoVA), Moskva 2017 [Европейские стандарты оценки. 8-е издание, 2016 - Eитореап Valuation Standards. Перевод Российского общества оценщиков, Европейская группа оценочных объединений (TEGoVA), Москва 2017]. 
[3] Gosudarstvennyy standart Respubliki Belarus' STB 52.0.02-2017: Otsenka stoimosti ob"yektov grazhdanskikh prav. Terminy i opredeleniya. Gosudarstvennyy komitet po standartizatsii Respubliki Belarus', Minsk 2017[Государственный стандарт Республики Беларусь СТБ 52.0.02-2017: Оценка стоимости объектов гражданских прав. Термины и определения. Государственный комитет по стандартизации Республики Беларусь, Минск 2017].

[4] Fomenko A.N.: Likvidatsionnayastoimost': osobennosti otsenki, sopostavleniye razlichnykh metodik. [in:] Sovremennyye tendentsii trekhstoronnego vzaimodeystviya, Bank-Zayemshchik-Otsenshchik, Universitet Sinergiya (Moskva), Assotsiatsiya otsenochnykh organizatsiy (Belarus'), Minsk, Belarus', 13.12.2017, 27 р. [Фоменко А.Н.: Аиквидациионая стоимость: особенности оценки, сопоставление различных методик. [в:] Современные тенденици трехстороннего взаимодействия, Банк-Заемщик-Оценщик, Университет Синергия (Москва), Ассоциация оценочных организаций (Беларусь), Минск, Беларусь, 13.12.2017, 27 с.].

[5] Hokhberh I.: Osoblyvosti otsinky obladnannya dlya tsiley kredytuvannya. [in:] Mizhnarodna naukovo-praktychna konferentsiya Praktychni aspekty otsinky ob'yektiv bankivs'koyi zastavy, Hromads'ka orhanizatsiya Vseukrayins'ke ob"yednannya, Ukrayins'ke tovarystvo otsinyuvachiv, L'Viv, 18-19 travnya 2017 [Гохберг I.: Особливості оцінки обладнання для ицілей кредитування. [в:] Міжнародна науково-практична конференція Практичні аспекти оцінки об'єктів банківської застави, Громадська організація Всеукраїнське об'єднання, Українське товариство оцінювачів, Иьвів, 18-19 травня 2017, 18 с.].

[6] Shabeka V., Yurenya S., Ignatkoviya G., Anufriyev P.: K voprosu o kharakteristike likvidnosti nazemnogo transporta. [in:] Prakticheskiye aspekty otsenki stoimosti otsenki stoimosti bankovskikh zalogov, Ukrainskoye obshchestvo otsenshchikov, L'viv, 18-19 maya 2017, 14 р. [Шабека В., Юреня С., Игнатковия Г., Ануфриев П.: К вопросу о характеристике ликвидности наземного транспорта. [в:] Практические аспекты оценки стоимости оценки стоимости банковскихзалогов, Украинское общество оценщиков, Пьвів, 18-19 мая 2017, 14 с.].

[7] Galasyuk V.V., Galasyuk V.V.: Opredeleniye likvidatsionnoy stoimosti po metodu Galasyukov (GMLV). Zarya, Dnepropetrovsk 2007 [Галасюк В.В., Галасюк В.В.: Определение ликвидационной стоимости по методу Галасюков (GMLV). Заря, Днепропетровск 2007].

[8] Государственный стандарт Республики Беларусь STB 52.3.01-2017: Otsenka stoimosti ob"yektov grazhdanskikh prav. Otsenka stoimosti kapital'nykh stroyeniy (zdaniy, sooruzheniy), ne zavershennykh stroitel'stvom ob'yektov, izolirovannykh pomeshcheniy, mashino-mest kak ob'yektov nedvizhimogo imushchestva. Государственный комитет по стандартизации Республики Беларусь, Минск 2017 [Государственный стандарт Республики 
Беларусь СТБ 52.3.01-2017: Оценка стоимости объектов гражданских прав. Оценка стоимости капитальных строений (зданий, сооружений), не завершенных строительством объектов, изолированных помещений, машино-мест как объектов недвижимого имущества. Государственный комитет по стандартизации Республики Беларусь, Минск].

[9] Korsunskiy D. [deputy general director of the Institute of Real Estate and Valuation Republican Unitary Enterprise], [on-line:] http://ino.by/about/ structure.html] [access: 12.04.2018].

[10] Sadlivskiy I. [expert in the work with auctions of RealConsulting LLC], [on-line:] http://www.expertiza.by/?212_2 [access: 12.04.2018].

[11] Koshevaya M., Shabeka V.L.: Klassifikatsiya ob'yektov nedvizhimosti dlya tseley otsenki stoimosti. [in:] IV Mezhdunarodnaya nauchno-prakticheskaya konferentsiya «Ekonomika, otsenka i upravleniye nedvizhimost'yui prirodnymi resursami», Vil'nyusskiy tekhnicheskiy universitet im. Gediminesa (28 marta 2014 goda, Vil'nyus, Litva), Belorusskiy gosudarstvennyy tekhnologicheskiy universitet (2-3 aprelya 2014 goda, Minsk, Belarus'), Vil'nyus, Minsk 2014, pp. 438-442 [Кошевая М., Шабека В.А.: Классификация объектов недвижимости для цеелей оценки стоимости. [в:] IV Международная научно-практическая конференция «Экономика, оценка и управление недвижимостью и природными ресурсами», Вильнюсский технический университет им. Гедиминеса (28 марта 2014 года, Вильнюс, Аитва), Белорусский государственный технологический университет (2-3 апреля 2014 года, Минск, Беларусь), Вильнюс, Минск 2014, с. 438-442].

\section{Teoretyczne i praktyczne aspekty określania wartości na potrzeby wymuszonej sprzedaży}

Streszczenie: Artykuł dotyczy kluczowych zagadnień związanych z szacowaniem wartości nieruchomości dla wymuszonej sprzedaży. Autor przedstawia zalecenia metodyczne dotyczące klasyfikacji różnych praw do nieruchomości jako przedmiotu wyceny. Przedstawiono materiały z ocen ekspertów i obserwacji empirycznych. Zaproponowano praktyczny model szacowania wartości na potrzeby wymuszonej sprzedaży.

Słowa

kluczowe: klasyfikacja wyceny, płynność, okres ekspozycji, okres realizacji celów wyceny 